\title{
Retroperitoneal Pheochromocytoma: A Rare Tumour Presenting as Ovarian Mass
}

\author{
BS Dhananjaya ${ }^{1}$, B Thanmaye ${ }^{2}$, G Sowmya ${ }^{3}$, M Naveen $^{4}$
}

\begin{abstract}
${ }^{1}$ Professor and HOD,${ }^{2}$ Post Graduate, ${ }^{3}$ Assistant Professor, Department of Obstetrics and Gynaecology,
${ }^{4}$ Associate Professor, Department of Surgery, Sri Siddhartha Medical College and Research Center, Agalkote, Tumakuru,
\end{abstract}

\section{Abstract}

A 40 year old patient suffering from vague pain in the abdomen since 1 month, came with CT scan of abdomen and pelvis showing large lobulated cystic lesion of $11 \times 10.6$ x $9.7 \mathrm{cms}$ suggesting ? Left ovarian neoplasm. On exploratory laparotomy uterus and ovaries were normal and a mass of $10 \times 9 \mathrm{~cm}$ was seen arising from the pelvis. The Histopathological report stated pheochromocytoma. The extra adrenal pheochromocytomas are rare tumors that originate from the chromaffin tissue of sympathetic nervous system.

Key words: Retroperitonial mass, pheochromocytoma, ovarian tumour.

\section{Introduction}

Pheochromocytomas (also known as paraganglioma are functional and non-functional tumours of the sympathetic nervous tissue. ${ }^{[1]}$ Catecholamines are secreted by functional pheochromocytomas, resulting in paroxysmal hypertension and palpitation. The majority of the pheochromocytomas are derived from chromaffin tissue of the adrenal medulla. ${ }^{[2]}$ Extraadrenal pheochromocytomas are rare tumours that originate from the chromaffin tissue of the sympathetic nervous system. ${ }^{[2]}$ In adults, pheochromocytomas are often called as $10 \%$ tumour because $10 \%$ occur along the diaphragm, while $10 \%$ of pheochromocytomas are extra- adrenal, $10 \%$ are bilateral, $10 \%$ are multiple, $10 \%$ are familial, $10 \%$ are malignant and $10 \%$ occur post operatively. ${ }^{[3]}$ Diagnosing pheochromocytomas is challenging due to their low incidence, and their clinical manifestations can be inconsistent. The present case report is an incidental finding of extra-adrenal nonfunctioning pheochromocytoma.

\section{Case report}

A 40 year old female was referred to Sri Siddhartha medial college with history of vague pain in the abdomen since 1 month with CT scan of abdomen and pelvis showing large lobulated solid cystic lesion of $11 \mathrm{x}$ $10.6 \times 9.7 \mathrm{~cm}$ arising from left adnexa. Few internal septations were seen within the lesion. The lesion is extending into infra-umbilical region superior to urinary bladder which was suspected to be left ovarian neoplasm. Routine hemogram and biochemical profile were within normal limits. CA125 was 5 units $/ \mathrm{ml}$. Patient was prepared for surgery. Upon exploration uterus and ovaries were found to be normal, a $10 \times 9 \mathrm{~cm}$ retroperitoneal mass was seen arising from the pelvis (Figure 1). It was clamped, cut and ligated and the mass was sent for histopathology report and it was consistent with pheochromocytoma showing trabecular arrangement of neoplastic cells with abundant cytoplasm (Figure 2). The post-operative period was uneventful.

\section{Discussion}

Pheochromocytomas are rare endocrine tumours that can present insidiously and remain undiagnosed until death. They may sometimes show onset of clear manifestations of catecholamines excess, most commonly as spells of headache, sweating, palpitation and hypertension. These tumours are no longer regarded as a uniform disease entity, but rather as a highly heterogenous group of chromaffin cell neoplasm with different ages of onset, secretory profiles, locations and potential for malignancy ascending to the underlying

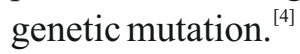

Patients with pheochromocytoma may develop complicated and potentially lethal cardiovascular and

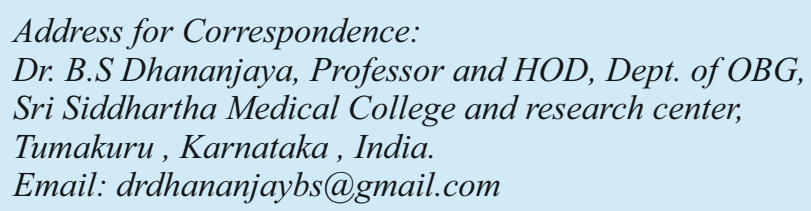




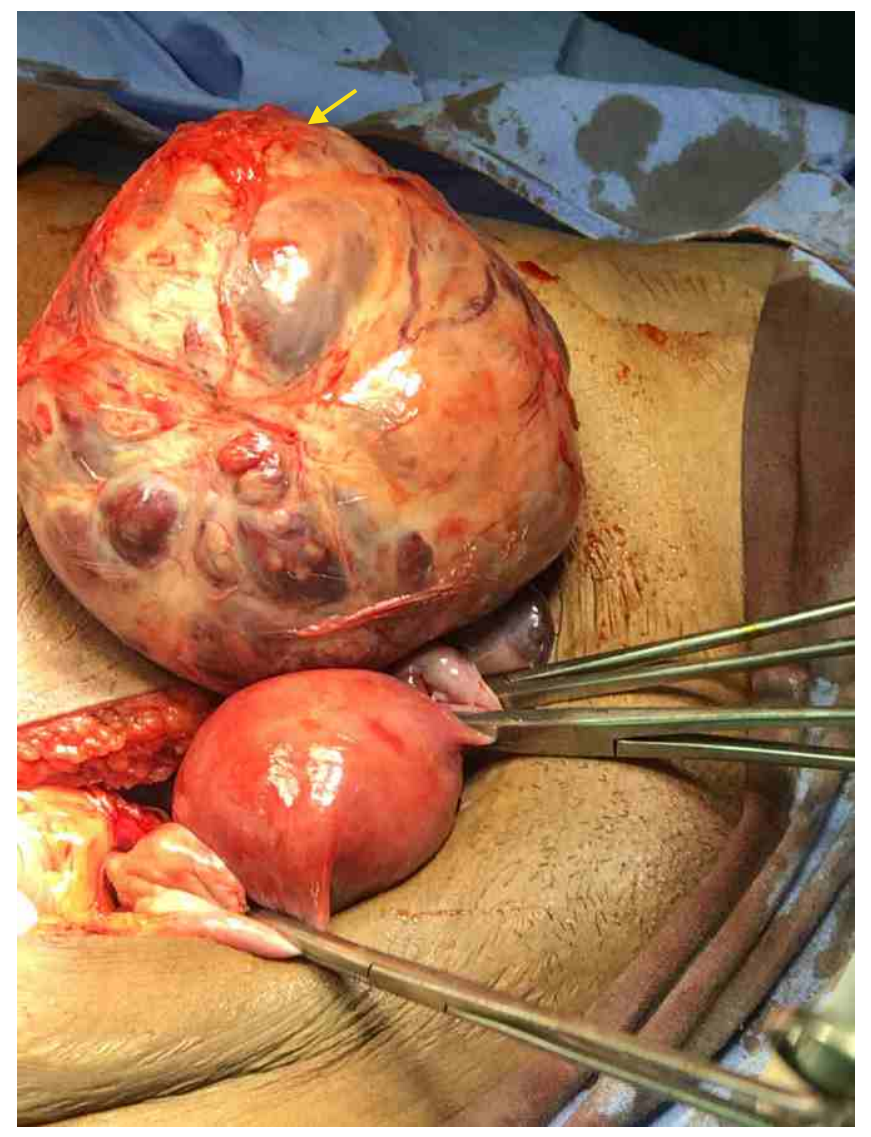

Figure 1: Gross features showing retroperitoneal mass (encircled) with normal uterus and ovaries.

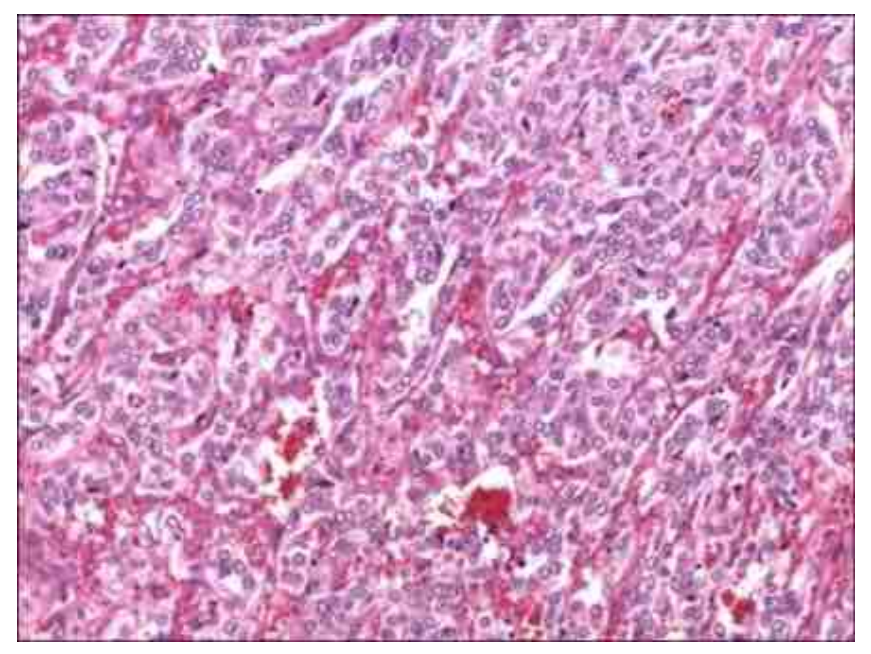

Figure 2: The $H$ and $E$ staining of the section shows neoplastic cells arranged in nests and trabecular pattern. cells have uniform round to oval nucleus with coarse chromatin and abundant eosinophilic cytoplasm.

other complications, especially during diagnostic or interventional procedures e.g., upon induction of anaesthesia or during surgery due to potent effect of paroxysmal release of catecholamines. ${ }^{[5]}$ Pelvic paraganglioma may present primarily to the gynaecologist and may be treated initially as preeclampsia or other pelvic tumours. ${ }^{[6]}$ The patient might present with vague pelvic symptoms and may not be forthcoming with the classical symptoms. ${ }^{[7]}$ The gynaecologist might miss these important clues leading to a potentially dangerous unplanned exploration. Suboptimal imaging may reveal the mass to be related to uterus and adnexa and careful thin section imaging with $3 \mathrm{D}$ reconstruction might reveal the exact anatomy of the mass.

Plasma and 24 hrs urine meta-nephrines might be normal which further adds to the confusion. Sudden intra-operative hypertension upon tumour manipulation may be the first hint towards this pathology, which was not evident in our case. A detailed history, physical examination and a complete laboratory and cardiac evaluation are essential in preparation of the patient with pheochromocytoma for surgery. Normalization of blood volume minimises the possibility of protracted hypotension or shock resulting from sudden diffuse vasodilation at the time of tumour removal and if catecholamine induced hyperglycaemia is found preoperatively, close monitoring must be initiated. ${ }^{[5]}$

Surgery is the only curative treatment while preoperative management has changed a little over the last 20 years, surgical procedures have evolved so that laparoscopic resection is the standard of care if feasible even for parauterine location. ${ }^{[3,4]}$ Radical surgical resection is the treatment of choice in giant extraadrenal pheochromocytomas. Due to the possibility of neoplasm recurrence and metastasis especially after incomplete surgical excision, periodic check-ups and follow-up is a must for the patients.

\section{Financial support and sponsorship: Nil Conflicts of interest: Nil}

\section{References}

1. Sheps SG, Jiang NS, Klee GG and van Heerden JA: Recent developments in the diagnosis and treatment of pheochromocytoma. MayoClin Proc 65: 88-95, 1990.

2. Elder EE, Elder G and Larsson C: Pheochromocytoma and functional paraganglioma syndrome: no longer the 10\% tumor. J Surg Oncol 89: 193-201, 2005.

3. Montemurro S, Ruggieri E, Maselli E, Zito AF, 
Chiumarulo F and Gargano G: A rare case of extraadrenal pheochromocytoma masquerading as an ovarian mass treated by laparoscopic surgery. Eur J Gynaecol Oncol 28: 491-496, 2007.

4. Da"rr R, Lenders JW, Hofbauer LC, et al. Pheochromocytoma; update on disease management. Ther Adv Endocrinol Metab. 2012;3(1):11-26.

5. Pacak K. Preoperative management of the pheochromocytoma patient. J Clin Endocrinol Metab. 2007;92(11):4069-79.

6. Kuscu E, Oktem M, Eroglu D, et al. Pelvic retroperitoneal paraganglioma mimicking an ovarian mass. Eur J Gynaecol Oncol. 2005;26(2):219-20.

7. Tohya T, Yoshimura T, Honda Y, et al. Unsuspected extraadrenal pheochromocytoma simulating ovarian tumor. Eur J Obstet Gynecol Reprod Biol. 1999;82(2):217-8.

8. Conzo G, Musella M, Corcione F, et al. Laparoscopic adrenalectomy, a safe procedure for pheochromocytoma. A retrospective review of clinical series. Int J Surg. 2013;11(2):152-6 